\title{
2. Über die Zeichen der Mathematik.
}

(Von Herrn Dr. Schellbach zu Berlin.)

$\bigcup_{m}$ den Gedanken gleich an einen bestimmten Gegenstand zu knüpfen, stellen wir hier die einfachen Rechnungsarten zusammen.

$$
\begin{aligned}
& \text { Addition } \quad a+b=c \mid \ldots 1 \text {. } \\
& \text { Subtraction }\left\{\begin{array}{ll}
c-a=b \\
c-b=a
\end{array}\right\} \ldots .2 . \\
& \text { Multiplication } a b=c \mid \ldots 4 \text {. } \\
& \text { Division } \\
& \left\{\begin{array}{l}
\frac{c}{a}=b \\
\frac{c}{b}=a
\end{array}\right\} \ldots 5 . \\
& \text { Logarithmirung } \begin{array}{l}
a \\
x
\end{array}=c(\ldots \pi \% \\
& \text { Extrahirung } \left.\quad a^{\frac{I}{c}}=b\right\} \ldots 8 \text {. } \\
& \text { Potenzirung } \left.\quad b^{c}=a\right) \ldots 9 \text {. }
\end{aligned}
$$

Das Zusammenfallen der Rechnungsarten (2.) und (3.) in die Subtraction, so wie (5.) und (6.) in die Division, erklärt sich aus der Gleichgültigkeit der Summanden und Factoren; in der dritten Gruppe, wo die Gröfsen $a, b, c$ verschiedene Bedeutung haben, sind auch die Rechnungsarten gesondert, welche durch sie bedingt werden.

Die Nothwendigkeit der Bezeichnungsweise des Potenzirens und Extrahirens zeigt sich darin, dafs jetzt weniger mit der Grundgröfse selbst operirt wird, als mit dem Operationszeichen, d. h. dals die Rechnung mit Potenzen und Wurzeln auf eine Rechnung mit ihren Exponenten zurückgebracht ist. Der Gedanke bietet sich von selbst dar, auch die Logarithmen durch eine schickliche Bezeichnung diesen Vortheil geniefsen zu lassen; daher ist statt der unvollstïndigen Formel

$$
\log a=c
$$


die Gleichung

entstanden.

$$
\underset{\substack{a \\ b}}{x}=c
$$

Die Wahl einer divisionsförmigen Bezeichnung der Logarithmen rechtfertigt sich wohl dadurch am besten, dafs

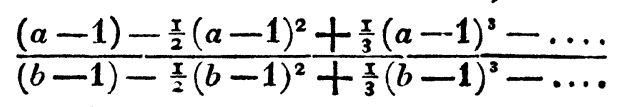

der Logarithmus von $a$ für die Basis $b$ ist.

Der Satz vom Modul wird dann auf folgende Weise geschrieben:

woraus sogleich folgt:

$$
\text { 10. } \quad \begin{array}{cc}
a & b \\
\underset{b}{b} & \underset{k}{x}
\end{array}=\underset{x}{a}
$$

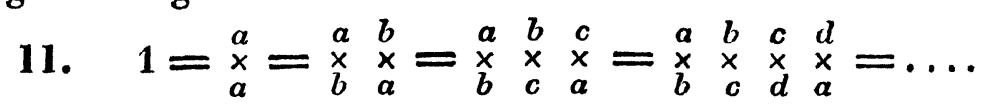

Nennt man in der Gleichung (\%) $a$ den Logarithmandus, $b$ die Basis und $c$ den Logarithmus, so läfst sich (11.) durch den Satz ausdrücken: In einem Producte verschiedener Logarithmen heben sich gleiche Basen gegen gleiche Logarithmanden auf.

In dieser Form ausgesprochen, prägt sich der Satz (10.) vom Modul dem Gediichtnifs auf der Stelle ein, weil er hier mathematischer erscheint, als in der gewöhnlichen Weise.

Man hat aufserdem folgende Verwandlungen:

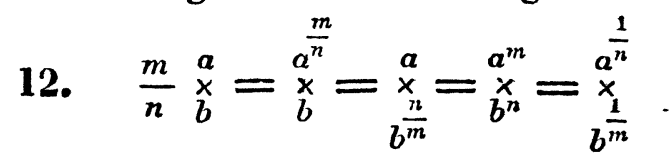

Auch hier zeigt sich der Vortheil einer divisionsförmigen Logarithmen-Bezeichnung deutlich.

So wie für die Multiplication und Division versehiedene Bezeichnungen beibehalten sind, weil sich manche Formeln auf die eine Weise geschickter darstellen lassen, als auf die andere, so kann man auch aus demselben Grunde für die Logarithmen noch eine zweite Bezeichnung einführen. Es ist nemlich ganz gleichbedeutend

eben so sei es mit

$$
\begin{array}{r}
a \times b \text { und } a \cdot b \\
\frac{a}{b} \text { und } a: b
\end{array}
$$

$$
\begin{aligned}
& a \\
& \underset{b}{b}
\end{aligned} \text { und } a: b
$$


Sind Logarithmand oder Basis zusammengesetzte Grölsen, so wird man die letzte Bezeichnung anwenden, also den Logarithmus von $a+b$ für die Basis $c$ schreiben:

$$
(a+b) \vdots c
$$

Dieser Bezeichnung wird man sich ebenfalls bedienen, wenn der Logarithmus von einem Logarithmus genommen werden soll; also drückt $(a \vdots b) \vdots c$

den Logarithmus von $a \vdots b$ für die Basis $c$ aus.

Durch diese Bezeichnung wird nun auch die formelle Auflüsung der Gleichung

$$
a^{b}=c
$$

den übrigen mathematischen Operationen analoger; denn so wie man sie sonst in Bezug auf a gewissermafsen multiplicationsweise auf lïste, durch

$$
a^{b \cdot \frac{1}{b}}=c^{\frac{1}{b}} \text { d. H. } a=c^{\frac{1}{b}} \text { : }
$$

so löst man sie jetzt in ähnlichem Sinne divisionsweise auf in Bezug auf $b$ durch

$$
\underset{a}{a^{b}}=\underset{a}{\stackrel{c}{a}}, \text { d. h. } b=\underset{a}{\stackrel{c}{a}}
$$

$$
\text { ร. } 2 .
$$

Der niichste Fortgang von den obigen 9 ersten Gleichungen ist durch die Gleichgültigkeit der Anzahl der Elemente $a, b, c$ gegeben. Sind diese ohne alle Beziehung zn einander, dann entwickelt sich aus den aufgestellten Gleichungen die Buchstabenrechnung. - Treten sie aber nur in die einfachste Beziehung der Aufeinanderfolge, so müssen wieder neue Zeichen gewählt werden, die sich an die schon vorhandenen anschliefsen. Hier sind nun zunächst folgende wesentliche Unterschiede der mathematischen Zeichen festzuhalten.

1. Entwicklungszeichen, Zeichen, die aus der Entwickelung der Mathematik selbst entstanden sind, ohne welche überhaupt kein wahrer Fortschritt dieser Wissenschaft möglich ist. Sind die ersten dieser Zeichen gesetzt, so ist die Form der folgenden auch schon bestimmt. Wegen der Einfachheit der ersten mathematischen Operationen wird der Willkür in der Bildung dieser Zeichen auch kein grofser Spielraum geblieben sein, und wir überzeugen uns bald von der Nothwendigkeit und Richtigkeit derselben; bauen wir also auf ihnen fort, so sind wir der 
Festigkeit unserer Grundlage versichert. Ein Blick auf die oben aufgestellte Tafel lehrt, dafs schon in der dritten Gruppe die Formen der beiden ersten wieder benutzt sind, wie z. B. bei den Bruch-Exponenten und Logarithmen; denn dafs sich hier die Bezeichnung durch $\log _{0}$ sehr fremdartig ausnehmen würde, leuchtet wohl hinlänglich ein.

2. A bkürzungszeichen, Zeichen, bei denen es nur darauf ankommt, das Wesentliche einer Formel, also das Verïnderliche, vom Unwesentlichen, dem Starren, Unveränderlichen, zu sondern, und in einem Bilde zusammenzufassen. Hier hat die Willkür schon bei weitem freieres Spiel. Soll z. B. der $k^{\text {te }}$ Binomialcoefficient der $n^{\text {ten }}$ Potenz ausgedrückt werden, so kann dies geschehen durch $n_{k}$, oder $(n, k)$, oder $\left(\begin{array}{l}n \\ k\end{array}\right)$, oder auf eine beliebige andere Weise, wenn nur die Elemente $n$ und $k$ in einem Ausdrucke abgesondert dargestellt werden. Solche Zeichen haben den Werth, dafs sie deutlich hervortreten lassen, was das Wesentliche eines Ausdrucks eigentlich ist; aber ihre Organisation stellt sich in den - einzelnen Fällen oft auf die mannigfaltigste Weise von selbst dar, kann daher auch nicht Gegenstand dieser Abhaudlung sein sollen. Unter diesen Zeichen können nicht leicht inconsequente vorkommen, wohl aber unter denen der ersten Art.

Bei der Bildung aller Zeichen ist der Grundsatz wichtig, nichts durch Buchstaben zu bezeichnen, was durch blofse Stellung, oder wohl gar schondurch Zahlen ausgedrückt werdenkann. Man giebt also die Folge der Coefficienten in Reihen immer durch Indices an, und bezeichnet Operationen nie durch das hingeschriebene Wort derselben, oder durch dessen erste Sylbe oder ersten Buchstaben; denn diese werden sich fast nie der Rechnung unterwerfen lassen, und gerade die höhern Theile der Mathematik sind eine Rechnung mit Rechnungszeichen.

Den ausgesprochenen Ansichten gemäls, gehen wir zur Bildung neuer Zeichen fort. Man bezeichnet eine Summe von $n$ gleichen Grölsen $a$ durch $n a$ oder

$$
a+a+a+a+\ldots+a=n a
$$

Demgemäls setze man nun die Summe der $n$ verschiedenen Grölsen

$$
\text { 1. } a_{0}+a_{1}+a_{2}+a_{3}+\ldots . .+a_{n-1}=n \mid a_{\sigma}
$$

Die Summenzahl $n$ giebt an, aus wie viel Gliedern die ganze Summe beCrelle's Journal d. M. Bd. XII. Hft. 1. 
steht. Dem Summenzeiger $\sigma$ müssen nach und nach alle ganze. Werthe von 0 bis $n-1$ beigelegt werden. Nimmt man die Reihe rückwïrts, so ist auch

$$
\text { 2. } a_{0}+a_{1}+a_{2}+a_{3}+\ldots .+a_{n-1}=n \mid a_{n-1-\sigma} .
$$

Wäre z. B. zu summiren

$$
0+1+2+3+\ldots+(n-1)=n \mid \sigma,
$$

so ist, mit Rücksicht auf (2.),

$$
n|\sigma=n|(n-1-\sigma)=n|(n-1)-n| \sigma, \quad n|2 \sigma=n(n-1), \quad n| \sigma=\frac{n(n-1)}{2} \text {. }
$$

An dem Gliede $n \mid(n-1)$ der obigen Gleichung, welches nichts anderes als $n(n-1)$ ist, zeigt sich, dafs die Summenzahl $n$ wieder zur Bedeutung eines blofsen Factors herabsinken kann, und eben darin ruht die Nothwendigkeit, die Summen auf die angegebene Weise zu bezeichnen; denn wenn sich ein Begriff aus einem allgemeineren entwickelt hat, so mufs er dessen Bestimmtheit noch mit an sich tragen, und es ist klar, dafs dies hier wirklich mit dem Begriffe der Summenzahl und dem allgemeineren des Factors der Fall ist.

\section{\$. 3.}

Diese Summenbezeichung ist also nur ein consequentes Erweitern der Bedeutung des Factors. Eine eben solche Erweiterung der Basis einer Potenz zur Basis einer Factorielle, hat zuerst auf die wissenschaftlichste Weise der Herausgeber dieser Zeitschrift, in einer Abhandlung des VII. Baudes derselben, eingeführt.

Wir entlehnen von ihm die Bezeichnung

$$
\text { 1. } \begin{aligned}
a(a+k)(a+2 k) \ldots(a+n k-k) & =(a,+k)^{n} \text { und } \\
\frac{1}{(a-k)(a-2 k)(a-3 k) \ldots(a-n k)} & =(a,+k)^{-n},
\end{aligned}
$$

und dehnen dieselbe auch auf das Product

$$
\text { 2. } a_{0} \alpha_{k} a_{2 k} a_{3 k} \ldots a_{n k-k}=a_{11,+k}^{n}
$$

aus. Eben so schreiben wir

$$
f(x) f(x+y) f(x+2 y) \ldots f(x+n y-y)=f^{n}(x,+y)
$$

und auch

$$
\frac{1}{\left(1-a_{0}\right)\left(1-a_{1}\right)\left(1-a_{2}\right) \ldots\left(1-a_{n-1}\right)}=\left(1-a_{n,+1}\right)^{-n} \text {. }
$$

Die Grundsiitze der Binomialcoefficienten lassen sich dann auf folgende Weise ausdrǜken:

3. $\quad \frac{m \cdot m-1 . m-2 \ldots . . m-n+1}{1.2 .3 \ldots . n}=\left(\frac{m,-1}{1,+1}\right)^{n}=\left(\frac{m,-1}{1,+1}\right)^{m-n}=\frac{m \cdot m-1}{1.2 .3 \ldots m-2 \ldots . n+1}$, 
4. $\left(\frac{m,-1}{1,+1}\right)^{n}+\left(\frac{m,-1}{1,+1}\right)^{n-1}=\left(\frac{m \cdot+1,-1}{1,+1}\right)^{n}$

5. $\left(\frac{n,-1}{1,+1}\right)^{m}\left(\frac{n-m,-1}{1,+1}\right)^{v}=\left(\frac{n,-1}{1,+1}\right)^{v}\left(\frac{n-v,-1}{1,+1}\right)^{m}$

6. $\left(\frac{n,-1}{1,+1}\right)^{m}\left(\frac{m,-1)}{1,+1}\right)^{n-v}=\left(\frac{n,-1}{1,+1}\right)^{v}\left(\frac{v,-1}{1,+1}\right)^{n-m}$

Die Formel (6.) erscheint hier vielleicht zuerst ; in ihr und in (5.) siud die angegebenen Vertauschungen der Elemente oft von Nutzen.

Wir schliefsen gleich noch ein brauchbares Zeichen an, um das blofse Vorkommen oder Auftreten von $n$ Gröfsen $a_{0}, a_{1}, a_{2}, \ldots a_{n-1}$ anzudeuten; dies geschehe nemlich durch

$$
\text { 7. } n ! a_{\delta}
$$

Dem Zeiger $\delta$ werden alle ganze Zahlen von 0 bis $n-1$ beigelegt. Also eine Function von $x_{1}, x_{1}, x_{2}, \ldots x_{n-1}$ wird ausgedrückt durch $f\left(n ! x_{j}\right)$. Diese Bezeichnung erspart oft viele Weitläufigkeiten.

Ferner bezeichnen wir die Combinationen ohne Wiederholungen zu je $m$ der Elemente $a_{0}, a_{1}, a_{2}, \ldots a_{n-1}$ durch

$$
\text { 8. }\left(m, n ! a_{\delta}\right)
$$

und die Combinationen mit Wiederholungen durch

$$
\text { 9. }\left[m, n ! a_{\delta}\right]
$$

Es finden bei dieser Bezeichnung bekanntlich folgende Formeln Statt:

10. $(m, s+1 ! n \pm s \mp \delta)=(m, s ! n \pm s \mp \delta)+n(m-1, s ! n \pm s \mp \delta)$

11. $[m, s+1 ! n \pm s \mp \delta]=[m, s ! n \pm s \mp \delta]+n[m-1, s+1 ! n \pm s \mp \delta]$

Die erste derselben heifst also: die Combinationen ohne Wieder. holungen zu $m$ Elementen aus den $s+1$ Elementen

oder auch

$$
n+s, n+s-1, n+s-2, \ldots n+1, n
$$

$$
n-s, n-s+1, n-s+2, \ldots n-1, n
$$

sind zusammengesetzt aus allen Combinationen dieser Art, denen das Glied $n$ fehlt, und dem Producte desselben mit den Combinationen zu $m-1$ Elementen, denen ebenfalls dieses Glied mangelt.

Ist in den beiden letzten Gleichungen $s=n$, und man wählt die untern Zeichen, so sind die Elemente nur $0,1,2,3, \ldots n$, und dann schreiben wir diese Gleichungen, mit Auslassung der Zeiger, ganz einfach

12. $(m, n+1)=(m, n)+n(m-1, n)$

13. $[m, n+1]=[m, n]+n[m-1, n+1]$ 


\section{4.}

Wir gehen jetzt zu einer Methode der Reihen-Entwickelung und der Summation über, déren Grundbegriffe uns einfacher und allgemeiner zu sein scheinen, als die der Differenzen-Rechnung, welche gewöhnlich zu diesem $Z_{\text {wecke angewandt wird. }}$

I. Hat man die Functionengleichung

$$
\text { 1. } \varphi(x)=f(x+y) \pm f(x)
$$

und multiplicirt sie mit $(\mp)^{n+1}$, nachdem man in ihr $x+n y$ statt $x$ gesetzt hat, so entsteht

$$
(\mp)^{n+1} \varphi(x+n y)=(\mp)^{n+1} f(x+(n+1) y)-(\mp)^{n} f(x+n y)
$$

Wird von dieser Gleichung die Summe nach $n$ genommen, so verwandelt sie sich in

$$
n\left|(\mp)^{\sigma+1} \varphi(x+\sigma y)=n\right|(\mp)^{\sigma+1} f(x+(\sigma+1) y)-n \mid(\mp)^{\sigma} f(x+\sigma y)
$$

Trennt man hier von der ersten Reihe der rechten Seite das letzte Glied ab, und von der zweiten Reihe das erste, so erhïlt man

$$
n(\mp)^{\sigma+1} \varphi(x+\sigma y)=
$$

$\left(n-1 \mid(\mp)^{\sigma+1} f(x+(\sigma+1) y)+(\mp)^{n} f(x+n y)-f(x)-\left(n-1 \mid(\mp)^{\sigma+1} f(x+(\sigma+1) y)\right.\right.$

Die beiden Reihen rechts heben sich nun geyen einander auf, und es bleibt

oder

$$
\text { 2. } \quad n\left\lfloor(\mp)^{\sigma+1} \varphi(x+\sigma y)=(\mp)^{n} f(x+n y)-f(x)\right.
$$

$\mp \varphi(x)+\varphi(x+y) \mp \varphi(x+2 y)+\ldots .+(\mp)^{n} \varphi(x+n y-y)=(\mp)^{n} f(x+n y)-f(x)$

$$
\text { II. Es sei 3. } \varphi(x)=f(x+y) \pm \psi(x) f(x)
$$

Man multiplicire diese Gleichung mit

$$
\psi(x+y) \psi(x+2 y) \psi(x+3 y) \ldots . \psi \psi(x+m y)=\psi^{m}(x+y,+y)
$$

so erhält man

$$
\psi^{m}(x+y,+y) \varphi(x)=\psi^{m}(x+y,+y) f(x+y) \pm \psi^{m+1}(x,+y) f(x)
$$

Diese Gleichung kann aufgefafst werden als

$$
V(m, x)=F(m-1, x+y) \pm F(m, x)
$$

und giebt dann, wenn man sie mit (1.) und (2.) vergleicht,

$$
n \mid(\mp)^{\sigma+1} V(m-\sigma, x+\sigma y)=(\mp)^{n} F(m-n, x+n y)-F(m, x)
$$

Wird nun $m=n-1$ gesetzt und das, was $V$ und $F$ bedeuten, so erhält man hieraus

4. $n \mid(\mp)^{\sigma+1} \psi^{n-1-\sigma}(x+y+\sigma y,+y) \varphi(x+\sigma y)=(\mp)^{n} f(x+n y)-\psi^{n}(x,+y) f(x)$ oder

$\mp \psi^{n-1}(x+y,+y) \varphi(x)+\psi^{n-2}(x+2 y,+y) \varphi(x+y) \mp \psi^{n-3}(x+3 y,+y) \varphi(x+2 y)+\ldots$

$$
\ldots .+(\mp)^{n} \varphi(x+n y-y)=(\mp)^{n} f(x+n y)-\psi^{n}(x,+y) f(x)
$$


Die Gleichung

$$
\text { 5. } \varphi(x)=\chi(x) f(x+y) \pm \psi(x) f(x)
$$

lärst sich eben so behandeln; denn multiplicirt man sie mit

so entsteht

$$
x^{m}(x-y,-y) \psi^{p}(x+y,+y)
$$

$$
\begin{array}{cc}
\chi^{m}(x-y,-y) \psi^{p}(x+y,+y) \varphi(x) \\
=\chi^{m+1}(x,-y) \psi^{p}(x+y,+y) f(x+y) \pm \chi^{m}(x-y,-y) \psi^{p+1}(x,+y) f(x) \\
\operatorname{der} \quad & V(m, p, x)=F(m+1, p-1, x+y) \pm F(m, p, x) .
\end{array}
$$

oder

und hieraus durch Vergleichung mit (1.) und (2.)

6. $n \mid(\mp)^{\sigma+1} V(m+\sigma, p-\sigma, x+\sigma y)=(\mp)^{n} F(m+n, p-n, x+n y)-F(m, p, x)$

Zur Versinnlichung dieser Formeln wählen wir einige Beispiele.

1) Es ist

oder

$$
x^{m}=x^{m}\left(\frac{x-1}{x-1}\right)=\frac{x^{m+1}}{x-1}-\frac{x^{m}}{x-1}
$$

Diese Gleichung verwandelt sich nach (2.) in

d. $h$.

$$
n \mid \varphi(m+\sigma)=f(m+n)-f(m)
$$

oder, wenn man mit $x^{m}$ dividirt:

$$
n \mid x^{m+\sigma}=\frac{x^{m+n}}{x-1}-\frac{x^{m}}{x-1}
$$

$$
\text { \%. } x^{n}+x^{1}+x^{2}+\ldots+x^{n-1}=n \mid x^{\sigma}=\frac{x^{n}-1}{x-1}
$$

2) Die Gleichung (4.) des $\$ .3$.

kann aufgefafst werden als

$$
\left(\frac{m+1,-1}{1,+1}\right)^{n}=\left(\frac{m,-1}{1,+1}\right)^{n}+\left(\frac{m,-1}{1,+1}\right)^{n-1}
$$

$$
f(m+1, n)=f(m, n)+f(m, n-1)
$$

und läfst sich danu in die drei Formen bringen:

$$
\begin{aligned}
& \text { 8. } f(m+1, n+1)=f(n, n+1)+f(m, n) \\
& \text { 9. } f(m, n+1)=f(m+1, n+1)-f(m, n) \\
& \text { 10. } f(m, n-1)=f(m+1, n)-f(m, n)
\end{aligned}
$$

deren Vergleichung mit (1.) und (2.) ergiebt:

$$
\begin{aligned}
& \text { 11. } \quad\left\lfloor(-)^{\sigma}\left(\frac{m+1,-1}{1,+1}\right)^{n+1+\sigma}=(-)^{k}\left(\frac{m,-1}{1,+1}\right)^{n+i}-\left(\frac{m,-1}{1,+1}\right)^{n}\right. \\
& k \mid\left(\frac{m+\sigma,-1}{1,+1}\right)^{n+1+\sigma}=\left(\frac{m+k,-1}{1,+1}\right)^{n+k}-\left(\frac{m,-1}{1,+1}\right)^{n} \\
& \text { 13. } k \mid\left(\frac{m+\sigma,-1}{1,+1}\right)^{n-1}=\left(\frac{m+k,-1}{1,+1}\right)^{n}-\left(\frac{m,-1}{1,+1}\right)^{n}
\end{aligned}
$$


Künftig wollen wir ein Glied, welches aus einem links vorhergehenden entsteht, wenn in diesem irgend ein Element, z. B. $k$, gleich Null gesetzt wird, durch $(k=0)$ bezeichnen. Dann schreiben wir z. B. die letzte dieser Gleichungen:

$$
\left(\frac{m,-1}{1,+1}\right)^{n-1}+\left(\frac{m+1,-1}{1,+1}\right)^{n-1}+\left(\frac{m+2,-1}{1,+1}\right)^{n-1}+\ldots+\left(\frac{m+k-1,-1}{1,+1}\right)^{n-1}=\left(\frac{m+k,-1}{1,+1}\right)^{n}-(k=0)
$$

3) Es ist

$$
\begin{aligned}
(a,+\alpha)^{p}(a+p a,+a)^{m-p} & =(a,+\alpha)^{m} \\
(a+m \alpha,+\alpha)^{p}(a+p a,+\alpha)^{m-p} & =(a+p a,+\alpha)^{m} \\
(a+m \alpha,+\alpha)^{p}(a,+\alpha)^{m} & =(a,+\alpha)^{m+p}
\end{aligned}
$$

Hier sollen $a$ und $\alpha$ ganz beliebige Elemente sein, $m$ und $p$ aber nur positive oder negative ganze Zablen. Bilden wir nun noch aus den entsprechenden Elementen $b$, und $\beta, n$ und $q$ drei ihnliche Gleichungen, so künnen diese mit den obigen auf folgende Weise zusammengestellt werden:

$$
\text { 14. } \begin{aligned}
\left\{\frac{(\alpha+m \alpha,+\alpha) p}{(b+n \beta,+\beta)^{q}} \pm \frac{(a+\alpha)^{p}}{(b,+\beta, q}\right\} \frac{(a+p \alpha,+\alpha)^{m-p}}{(b+q \beta,+\beta)^{n-q}} & =\frac{(a+p \alpha,+\alpha)^{m}}{(b+q \beta,+\beta)^{n}} \pm \frac{(a,+\alpha)^{m}}{(b,+\beta)^{n}} \\
\text { 15. }\left\{\frac{\alpha+m \alpha+\alpha}{(b+n \beta,+\beta)^{q}} \pm 1\right\} \frac{(a,+\alpha)^{m}}{(b,+\beta)^{n}} & =\frac{(a,+\alpha)^{m+p}}{(b,+\beta)^{n+q}} \pm \frac{(a,+\alpha)^{m}}{(b,+\beta)^{n}}
\end{aligned}
$$

Diese Gleichungen lassen sich auffassen als $V(a, b)=F(a+p a, b+q \beta) \pm F(a, b)$ und $V(m, n)=F(m+p, n+q) \pm F(m, n)$ und werden durch Vergleichung mit (1.) und (2.) summirt. Aus (14.) erhalten wir z. B. für $m=n, p=1, q=-1$

$$
\begin{aligned}
& \text { 16. } \frac{(a,+\alpha)^{n-1}}{(b,+\beta)^{n+1}}+\frac{(a-\alpha,+\alpha)^{n-1}}{(b+\beta,+\beta)^{n+1}}+\frac{(a-2 \alpha+\alpha)^{n-1}}{(b+2 \beta,+\beta)^{n+1}}+\cdots+\frac{(a-k \alpha+\alpha,+\alpha)^{n-1}}{(b+k \beta-\beta,+\beta)^{n+1}}= \\
& k \mid \frac{(a-\alpha \sigma,+\alpha)^{n-1}}{(b+\beta \sigma,+\beta)^{n+1}}=\frac{(a-k \alpha,+\alpha)^{n}}{n(\alpha \beta-n \alpha \beta-a \beta-b \alpha)(b+k \beta,+\beta)^{n}}-(k=0)
\end{aligned}
$$

Bei diesen Summationen kommt es nur darauf an, die Elemente in den Gleichungen (14.) und (15.) so zu wählen, dals die Summenzeiger aus den eingeklammerten Theilen der linken Seite verschwinden. Die Gleichungen (14.) und (15.) sind sehr allgemein; aus ihnen fliefsen auch leicht die Reihen (11.), (12.) und (13.), so wie noch viele andere.

4) Setzt man

$$
x y=z
$$

wo $y$ und $z$ Functionen von $x$ sein mügen, so erbïlt man durch $n$ maliges Differenziren nach $x$, und Multipliciren mit $\frac{x^{n-1}}{n}$ die Gleichung

$$
\frac{x^{n-1}}{n} \frac{\partial^{n} z}{\partial x^{n}}=x^{n-1} \frac{\partial^{n-1} y}{\partial x^{n-1}}+\frac{x^{n}}{n} \frac{\partial^{n} y}{\partial x^{n}}
$$

welche aufgefafst worden kann als 


$$
\varphi(n)=f(n-1)+\psi(n) f(n), \ldots
$$

und dann durch Vergleichung mit (3.) und (4.) folgende Reihe giebt:

$$
z=k \mid(-)^{\sigma} \frac{x^{1+\sigma}}{(1,+1)^{1+\sigma}} \frac{\partial^{1+\sigma} z}{\partial x^{1+\sigma}}+(-)^{k} \frac{x^{k+1}}{(1,+1)^{k}} \frac{\partial^{k} y}{\partial x^{k}}
$$

5. Wir haben bis jetzt die Auflösung der Gleichung (1.) und (3.) nur benutzt, um $\Phi x$ in eine Reihe zu entwickeln; sie diene zugleich aber auch dazu, die Form von $f_{x}$ durch $\varphi_{x}$ und $\psi x$ zu bestimmen, oder, was dasselbe ist, zur Integration von Differenzengleichungen.

Bekanntlich sind von den Differenzengleichungen

die Integrale

$$
y_{1}=R y+Q \text { und } \Delta y+P y=Q
$$

$$
y=\left[R_{x-1}\right] \Sigma \frac{Q}{\left[R_{x}^{x+1}\right]} \text { und } y=\left[1-P_{x-1}\right]^{x} \Sigma \frac{Q}{\left[1-P_{x}{ }^{x+1}\right.}
$$

Diese beiden Differenzengleichungen sind aber nichts anderes, als

$$
f(x+h)-\psi(x) f(x)=\varphi(x) \text { und } f(x+h)-(1-\psi x) f(x)=\varphi x
$$

und nach der Formel (4.) erhält man hieraus durch eine leichten Änderung der Werthe der Elemente

und

$$
f(h x)=\psi^{x}(0,+h)\left\{x \mid \frac{\varphi(h \sigma)}{\psi^{0+1}(0,+h)}+f 0\right\}
$$

$$
f(h x)=(1-\psi(0,+h))^{x}\left\{x \mid \frac{\varphi(h \sigma)}{1-\psi^{\prime}(0,+h)^{\sigma+1}}+f 0\right\}
$$

wo $f 0$ die zum Integral zu fügende Constante ist.

Für $h=1$ fallen diese Ausdrücke mit den obigen Integralen zusammen. Es scheint mir übrigens vortheilhaft, diese Integrationen und die obigen Reihenentwickelungen in einen unmittelbarern Zusammeuhang zu bringen, als gewöhnlich geschieht.

\section{§. 5.}

Ist $\phi(n, k)$ eine Function der Gröfsen $n$ und $k$ von der Beschaffenheit, dafs

$$
\text { 1. } \varphi(n+1, k+1)=\varphi(n+1, k)+\varphi(n, k)
$$

und dafs sie für negative Werthe von $n$ und solche, die gröfser als $k$ sind, verschwindet, so folgt aus diesen Annahmen, wenn man erst $n=-1$ und dann $n=k$ setzt,

Hat man nun die Gleichung

$$
\text { 2. } \varphi(0, k+1)=\varphi(0, k)
$$

3. $\varphi(k+1, k+1)=\varphi(k, k)$

$$
\text { 4. } f(x)=f(x+y) \pm f(x+z)
$$


und multiplicirt sie mit $( \pm)^{n} \varphi(n, k)$, nachdem man in ihr $x+(z-y) n$ statt $x$ gesetzt hat, so erhält man

$$
\begin{gathered}
\text { 5. }( \pm)^{n} \varphi(n, k) f(x+(z-y) n) \\
=( \pm)^{n} \varphi(n, k) f(x+y+(z-y) n)+( \pm)^{n+1} \varphi(n, k) f(x+y+(z-y)(n+1))
\end{gathered}
$$

Bezeichnet man der Kürze wegen $f(x+(z-y) n)$ durch $V(n)$ und $f(x+y+(z-y) n)$ durch $F(n)$, und denkt sich dann $n$ durch die ganzen Zahlen von 0 bis $n$ veränderlich, so verwandelt sich (5.) in $\left(n+1 \mid( \pm)^{\sigma} \varphi(\sigma, k) V(\sigma)=\left(n+1 \mid( \pm)^{\sigma} \varphi(\sigma, k) F(\sigma)+\left(n+1 \mid( \pm)^{\sigma+1} \varphi(\sigma, k) F(\sigma+1)\right.\right.\right.$ Wird nun von der ersten Reihe der rechten Seite das erste Glied abgesondert, und von der letzten das letzte, so entsteht mit Rücksicht auf (1.) und (2.)

$$
\begin{aligned}
& \quad\left(n+1 \mid( \pm)^{\sigma} \varphi(\sigma, k) V(\sigma)\right. \\
& =\varphi(0, k) F(0)+n\left|( \pm)^{\sigma+1} \varphi(\sigma+1, k) F(\sigma+1)+n\right|( \pm)^{\sigma+1} \varphi(\sigma, k) F(\sigma+1)+( \pm)^{n+1} \varphi(n, k) F(n+1) \\
& =\varphi(0, k+1) F(0)+n \mid( \pm)^{\sigma+1}\{\varphi(\sigma+1, k)+\varphi(\sigma, k)\} F(\sigma+1)+( \pm)^{n+1} \varphi(n, k) F(n+1) \\
& =\varphi(0, k+1) F(0)+n \mid( \pm)^{\sigma+1} \varphi(\sigma+1, k+1) F(\sigma+1)+( \pm)^{n+1} \varphi(n, k) F(n+1) \\
& =\varphi\left(n+1 \mid( \pm)^{\sigma} \varphi(\sigma, k+1) F(\sigma)+( \pm)^{n+1} \varphi(n, k) F(n+1)\right.
\end{aligned}
$$

oder

$$
\begin{gathered}
\text { 6. } \quad\left(n+1 \mathbf{J}^{\sigma}( \pm) \varphi(\sigma, k) f(x+(z-y) \sigma)\right. \\
=\left(n+1 \mid( \pm)^{\sigma} \varphi(\sigma, k+1) f(x+y+(z-y) \sigma)+( \pm)^{n+1} \varphi(n, k) f(x+y+(z-y)(n+1))\right.
\end{gathered}
$$

Verschwindet für irgend einen Werth ron $n$, den wir durch $r$ bezeichnen wollen, das letzte Glied $\varphi(r, k) f(x+z+z(z-y) r)$ dieser Gleichung, so erhalten wir

7. $\left(r+1 \mathbf{I}( \pm)^{\sigma} \varphi(\sigma, k) f(x+(z-y) \sigma)=\left(r+1 \mid( \pm)^{\sigma} \varphi(\sigma, k+1) f(x+y+(z-y) \sigma)\right.\right.$ Ist aber $n=k$, so kann, vermöge der Bedingung (3.), auch das letzte Glied der Formel (6.) mit in die Reihe der rechten Seite aufgenommen werden, und es ergiebt sich daraus dann

$$
\text { 8. }\left(k+1 \mid( \pm)^{\pi} \sim(\sigma, k) f(x+(z-y) \sigma)=\left(k+2 \mid( \pm)^{\sigma} \varphi(\sigma, k+1) f(x+y+(z-y) \sigma)\right.\right.
$$

Die Gleichungen (\%.) und (8.) lehren, dafs man die Gröfse $x$ immer um ein $y$ und die Grölse $k$ um die Einheit vermehren oder vermindern kann, bis man, wenn dies $n$ mal geschehen ist, zu den Formeln gelangt:

9. $\left(r+1 \mid( \pm)^{\sigma} \varphi(\sigma, k) f(x+(z-y) \sigma)=\left(r+1 \mid( \pm)^{\sigma} \varphi(\sigma, k \pm n) f(x \pm n y+(z-y) \sigma)\right.\right.$ 10. $\left(k+1 \mid( \pm)^{\sigma} \varphi(\sigma, k) f(x+(z-y) \sigma)=\left(k \pm n+1 \mid( \pm)^{\sigma} \varphi(\sigma, k \pm n) f(x \pm n y+(z-y) \sigma)\right.\right.$ welche mit Rücksicht auf die Bedingungen, denen die Function $\varphi$ unterworfen ist, für $k=0$ und $\varphi(0,0)=c$ in die folgenden übergehen :

11. $\quad c f(x)=\left(r+1 \mid( \pm)^{\sigma} \varphi(\sigma, n) f(x+n y+(z-y) \sigma)\right.$

12. $\quad c f(x)=\left(r+1 \mid( \pm)^{\sigma} \varphi(\sigma, n) f(x-n y+(z-y) \sigma)\right.$

13. $\quad c f(x)=\left(n+1 \mid( \pm)^{\sigma} \varphi(\sigma, n) f(x+n y+(z-y) \sigma)\right.$ 
In der Gleichung (10.) kann ein negatives $n$ bei der Annahme $k=0$ nicht Statt finden, weil man nicht weifs, was eine negative Summenzahl bedeutet, wohl aber bei der Annahme $n=k$, wodurch man

$$
\left(k+1 \mid( \pm)^{\sigma} \varphi(\sigma, k) f(x+(z-y) \sigma)=c f(x-k y)\right.
$$

findet, welche Gleichung aber, für $x+k y$ statt $x$, mit (13.) identisch wird. Eben so entsteht aus (9.) eine mit (11.) identische Gleichung, wenn man für den negativen Werth von $n, k=n$ setzt.

Aus der Gleichung (4.) des $\$ 3$. ist ersichtlich, dafs die Binomialcoefficienten unter der in (1.) aufgeführten Function verstanden werden können. Wir schliefsen daher aus (13.), dafs sich die Functionengleichung

$$
\text { 14. } f(x)=f(x+y) \pm f(x+z)
$$

immer bin o mis ch entwickeln läfst, so dafs'

$$
\begin{gathered}
\text { 15. } f(x)=\left(n+1 \mid( \pm)^{\sigma}\left(\frac{n,-1}{1,+1}\right)^{\sigma} f(x+n y+(z-y) \sigma)=\right. \\
f(x+n y) \pm\left(\frac{n,-1}{1,+1}\right) f(x+n y-y+z)+\left(\frac{n,-1}{n,+1}\right)^{2} f(x+n y-2 y+2 z) \pm\left(\frac{n,-1}{1,+1}\right)^{2} f(x+n y-3 y+3 z) \\
+\ldots .+( \pm)^{n} f(x+n z)
\end{gathered}
$$

Verschwindet aber fir irgend einen Werth von $r$ das Glied $f(x+z+(z-y) r)$, so erhält man, nach (11.) und (12.), auch noch

$$
\begin{aligned}
& \text { 16. } f(x)=\left(r+1 \mid( \pm)^{\sigma}\left(\frac{n,-1}{1,+1}\right)^{\sigma} f(x+n y+(z-y) \sigma)\right. \\
& \text { 17. } f(x)=\left(r+1 \mid( \pm)^{\sigma}\left(\frac{-n,-1}{1,+1}\right)^{\sigma} f(x-n y+(z-y) \sigma)\right.
\end{aligned}
$$

Ganz auf dieselbe Weise hätte man auch die noch allgemeinern Functionen

$$
\text { 18. } \varphi(n+1, k+1)=\varphi(n+1, k)+a \varphi(n, k)
$$

und

$$
\text { 19. } f(x)=f(x+y) \pm a f(x+z)
$$

mit einander combiniren können, wo $a$ als ein constanter Factor angenommen wird. Die Yergleichung von (18.) mit der Gleichung (12.) des §. 3. lehrt, dafs unter dieser Form die Combinationen ohne Wiederholungen begriffen sind, dafs sich also eine Gleichung wie (19.) in eine Reihe entwickeln läfst, deren Coefficienten aus solehen Combinationen bestehen.

(Der Schlufs folgt im nächsten Hefte.) • 